\title{
Choline PET/CT and intraoperative management of primary brain tumors. New insights for contemporary neurosurgery
}

\author{
Ignazio G. Vetrano ${ }^{1} \cdot$ Riccardo Laudicella $^{2} \cdot$ Pierpaolo Alongi $^{3}$
}

Received: 1 September 2020 / Accepted: 21 October 2020 / Published online: 12 November 2020

(c) Italian Association of Nuclear Medicine and Molecular Imaging 2020

Maximal safe resection represents the main aim of brain tumor surgery. Increasing the extent of tumor removal but preserving neurologic functions is the basilar concept of contemporary neurosurgery. This concept is particularly true for primary brain tumors, mainly gliomas. The differences in the clinical presentation, tumor location, biological behavior, and bio-molecular characteristics determine the need for a strictly individualized approach to a patient harboring a glioma. This necessity demanded, during the last years, continuous improvement of pre- and intra-operative imaging techniques, to optimize the surgical goals. Since its introduction, magnetic resonance imaging (MRI) has acquired the main diagnostic role in preoperative diagnosis, and also as intra-operative surgical guidance. MRI is, therefore, the modality of choice for the diagnosis and follow-up of glioma patients. Whereas this technique provides excellent anatomic details, MRI is not always able to supply exact functional information about tumor's grading and proliferative activity, despite advanced MRI sequences are becoming widely used to characterize altered metabolism. Namely, in some conditions as intra-tumoral bleeding, the pivotal role of MRI could be limited: although rarely, the primary diagnosis based on MRI-only, could be difficult to achieve. This aspect acquires particular relevance in the intra-operative setting: thanks to continuous technological advancements, neurosurgery has greatly benefited from a strict interaction with imaging modalities. The availability of intra-operative tools for imaging-guided surgery, determining a more robust

Pierpaolo Alongi

alongi.pierpaolo@gmail.com

1 Department of Neurosurgery, Fondazione IRCCS Istituto Neurologico Carlo Besta, Milan, Italy

2 Nuclear Medicine Unit, Department of Biomedical and Dental Sciences and Morpho-Functional Imaging, University of Messina, 98125 Messina, Italy

3 Unit of Nuclear Medicine, Fondazione Istituto G. Giglio, Contrada Pietrapollastra-Pisciotto, 90015 Cefalu, Italy accuracy in spatial orientation, has contributed to improving the neurosurgeon's ability to perform surgical procedures that minimize the risks for the patients. Neuronavigation, based on pre-operative imaging (mainly MRI), allow realtime visualization of tridimensional reconstructions from intracranial structures, guiding, therefore, craniotomy marking and planning the route of surgical procedure or biopsy. However, the tumor tissues delineated by MRI may not always correlate with the neoplasm portion having the highest grade potential. This could affect the identification of the optimal biopsy target, or impair the extent of resection. In neuro-oncology, positron emission tomography (PET) represents a valuable tool for the discrimination between low- from high-grade gliomas, and to distinguish between tumor recurrence and radionecrosis. However, the use of nuclear medicine techniques in neuro-oncological surgery is probably under-represented if compared to the wide use of PET in the oncological field of other organs and structures. This is mainly due to a historical question: $18 \mathrm{~F}$-fluorodeoxyglucose (FDG) was the first radiotracers used for brain tumors imaging, but the high avidity for the normal cortex, or basal ganglion region, has reduced, for a long time, its application in neuro-oncology. Namely, the normal brain tissue has the highest glucose metabolic rate of any other healthy tissue in the body; therefore, a technique depending on the metabolism of glucose may not be optimal to detect primary brain tumors. Further, the usefulness of FDG in tumor delineation, due to high uptake in the normal cortex and low uptake in low-grade gliomas, is particularly limited for cortical tumors. However, the introduction of radiolabeled amino acids, such as $18 \mathrm{~F}$-fluoroethyltyrosine (FET) and 11C-methionine (MET), has progressively shifted this paradigm to a growing application of nuclear medicine in gliomas patients' assessment. The uptake of FET and MET in the normal parenchyma is low, thus radiolabeled amino acids can identify primary brain tumors, highlighting a different intra-lesional uptake compared to the surrounding normal brain. Differently from FDG, amino acid PET 
imaging more accurately identifies infiltrating regions of tumor extending beyond the MRI contrast-enhancing lesion, thus helping to distinguish among oedema, normal brain, and neoplasm [1]. Although MR imaging provides useful morphological information, the contrast enhancement is unspecific for the tumor grading and advanced specialized MR metabolic techniques (i.e., spectroscopy, iron-oxide particles contrast agents) are not widely employed, do not always offer high-spatial-resolution and have operator/scanner variability. In addition, conventional MR suffers from compromised specificity in tumor surveillance in the presence of treatment-related changes. Moreover, previous data suggest that, in recurrent high-grade gliomas, intraoperative fluorescent dyes and amino acid PET both may demarcate larger tumor volumes than paramagnetic enhanced MRI [2]. In both low- and high-grade (WHO grade III/IV) gliomas, amino acid PET provide additional information to conventional MRI about tumor extent and biology: most grade II gliomas are non-enhancing, and the delineation of tumor borders by amino acid PET is superior to standard MRI in non-contrast-enhancing gliomas, as well as in contrastenhancing tumors [3]. Dynamic PET acquisition protocol using 18F-FET is becoming widely used in the clinical scenario and approved by European guidelines being able to add further crucial information for grade differentiation and evaluation of recurrences in gliomas [4].

More recently, other widespread and easily available radiotracers such as choline (CHO) analogues have demonstrated similar results [5]. Hara and coworkers introduced, in 1997, the use of carbon-11 (11C) CHO as radiotracers for PET imaging [6]. 11C-CHO is biochemically indistinguishable from native $\mathrm{CHO}$; however, the short decay halflife of the $11 \mathrm{C}(t / 2=20 \mathrm{~min})$ has limited its use to centers equipped with an on-site cyclotron. Subsequently, longerlived fluoro-18 labeled CHO derivates were developed, such as $18 \mathrm{~F}$-fluorometilcholine and 18F-fluoroethylcholine, exploiting the favorable half-life of $18 \mathrm{~F}(t / 2=110 \mathrm{~min})$ and better radiotracer distribution on the time improving signal/ noise ratio.

Radiolabelled $\mathrm{CHO}$ has been investigated for brain imaging, because of its very low physiological distribution rate in normal white and grey matter, except for choroid plexus and pineal gland, thus facilitating the identification of brain lesions. Choline is an essential protein that serves as an extrinsic substrate for the synthesis of phosphatidylcholine, which is a major constituent of the cell membrane. Phosphorylation by choline kinase constitutes an important step in the incorporation of choline into phospholipids, which is relevant for cell viability. In cancer, there is often an increase in the cellular transport and phosphorylation of choline, as well as an increase in the expression of choline kinase enzyme. Differently from FET and similarly to FLT, Choline-uptake is dependent on BBB damage and, therefore, it should be preferentially used in HGG. Therefore, several reports about the role of CHO-PET in diagnosis and management of brain tumors have been presented for the primary diagnosis of glioblastoma after intratumoral hemorrhage, or in pediatric astrocytomas $[4,5]$. However, we consider that CHO-PET could play a relevant role as intra-operative guidance for surgical procedures, and preliminary and interesting papers support our aim.

One of the first reports about the use of CHO-labeled radiotracers date up to 2003 when Hara et al. presented a series of gliomas in which they compared 18-F Cho with 11C-Cho [7]. The areas of increased uptake were then selected as a target for biopsy (whereas PET imaging where not integrated into a neuronavigator system). They found that the uptake of both radiotracers was always low in low-grade gliomas, and high in the high-grade tumors. The tumor/normal $(\mathrm{T} / \mathrm{N})$ ratio of 18F-choline was 10.5:12 in anaplastic astrocytoma and 13.2:21 in glioblastoma. However, the 18F-choline yielded superior results compared with $11 \mathrm{C}$-choline concerning the $\mathrm{T} / \mathrm{N}$ ratio.

A recently just published review exactly evaluate the possible application of prostate radiopharmaceutical agents, as Cho and PSMA, in glioma assessment describing the performances of this technique in gliomas [8]. Globally, the authors affirmed that 18F- and 11C-CHO PET are useful as an adjunctive guide to the surgical approach, to identify the most malignant areas for stereotactic sampling. Since that paper, we have waited 14 years to evaluate a more extensive work about the integration of metabolic imaging with MRI as surgical guidance in the operating room. Namely, Grech et al. presented in 2017 a series of patients harboring gliomas that underwent a biopsy or resection [9]. Interestingly, the authors integrated presurgical 18F-methylcholine PET and MR spectroscopy (MRS) with intraoperative neuronavigation, to guide surgical biopsy and tumor sampling of brain gliomas. Based on such imaging integration, the regions of high and low choline uptake were represented as color-coded spheres, which represented the target for tissue sampling. Their results were really interesting: the coregistration of metabolic imaging allowed identifying areas of high mitotic activity in a suspected low-grade tumor, according to conventional MRI. More recently, in 2020, Villena Martin et al. reported two adjunctive cases of multicentric glioblastoma, in which MRI and Cho-PET were performed to define the target of the biopsy in the most CHO-avid area [5]. They used the metabolic information acquired by PET to guide the MRS voxel placement in a tumor region of the suspected highest grade, confirming the value of the co-registration of PET and MRI, loaded into the neuronavigator, as intraoperative guidance, especially in case of multicentric tumors with areas of different paramagnetic contrast enhancement. All these findings appear relevant in the era of molecularbased neuro-oncology: the advent of molecular markers, as 
standardized by 2016 WHO classification of brain tumors [10], has shifted the landscape in glioma treatment towards more personalized therapies. In the restaging process, $\mathrm{Li}$ et al. showed that the sensitivity and specificity of $11 \mathrm{C}-\mathrm{CHO}$ $\mathrm{PET} / \mathrm{CT}$ in detecting tumor recurrence were 100 and $70 \%$, whereas those of MRI were 83.3 and $60 \%$, respectively; moreover, the SUVmax and LNR of 11C-CHO uptake strongly correlated with the overall and progression-free survival [12]. Tatenaka et al. showed that a T/N ratio of Choline was significantly higher in Glioblastomas than in readionecrosis [13].

Cho-PET could enable noninvasive imaging of cellular metabolism relevant to proliferation and can detect regions of more highly active tumor [11] at staging and in the post-treatment evaluation. Furthermore, Cho-PET is today a widely diffuse in most nuclear medicine departments, permitting quick scheduling of examinations, being more cost-effective in comparison to other aminoacidic radiotracers. However, the use of this radiopharmaceutical agent is still not approved in the clinical scenario for brain tumors and the current application demonstrated in the scientific literature are limited to off-label use. PET imaging is not yet fully widespread and not fully used, and MR imaging is well-established and easier to get. Furthermore, where available, PET imaging mainly consists of FDG/prostate-cancer scan and only a few centers offer the possibility of studying brain tumors for both organizational, cost and authorization problems. Further, it is necessary to train neuro-oncology PET experts thus improving a wide modality diffusion and effectiveness, potentially translating in this scenario the use of Choline.

Starting from these considerations, and considering the role of Cho-PET as underestimated respect to all its potentialities in neuro-oncology, we believe that combined PET and MRI hybrid imaging systems will further enhance bioimaging-based brain tumor surgery. Whereas the experiences at date are limited, $\mathrm{CHO}$ is an effective radiotracer with potential optimal performances in gliomas. CHO-PET could become progressively an essential part of the neurosurgical armamentarium.

Author contributions IGV and PA equally contributed to conception and design of the article. IGV, RL and PA: Final approval of the version to be published. IGV, RL and PA: Drafting of the article or critical revision for important intellectual content.

Data availability Not applicable.

Code availability Not applicable.

\section{Compliance with ethical standards}

Conflict of interest All the authors declare that they have no conflict of interest related to this spotlight.

Ethics approval Not applicable.

Consent to participate Not applicable.

Consent for publication Not applicable

\section{References}

1. Torii K, Tsuyuguchi N, Kawabe J et al (2005) Correlation of amino-acid uptake using methionine PET and histological classifications in various gliomas. Ann Nucl Med 19:677-683. https ://doi.org/10.1007/BF02985116

2. Buchmann N, Kläsner B, Gempt J et al (2016) 18F-FluoroethylL-Thyrosine positron emission tomography to delineate tumor residuals after glioblastoma resection: a comparison with standard postoperative magnetic resonance imaging. World Neurosurg 89:420-426. https://doi.org/10.1016/j.wneu.2016.02.032

3. Laudicella R, Iagaru A, Minutoli F et al (2020) PET/MR in neuro-oncology: is it ready for prime-time? Clin Transl Imaging 8:233-235

4. Law I, Albert NL, Arbizu J et al (2019) Joint EANM/EANO/ RANO practice guidelines/SNMMI procedure standards for imaging of gliomas using PET with radiolabelled amino acids and [18 F]FDG: version 1.0. Eur J Nucl Med Mol Imaging 46:540-557. https://doi.org/10.1007/s00259-018-4207-9

5. Villena Martín M, Pena Pardo FJ, Jiménez Aragón F et al (2020) Metabolic targeting can improve the efficiency of brain tumor biopsies. Semin Oncol 47:148-154. https://doi.org/10.1053/j. seminoncol.2020.04.007

6. Hara T, Kosaka N, Shinoura N, Kondo T (1997) PET imaging of brain tumor with [methyl-11C]choline. J Nucl Med 38:842-847

7. Hara T, Kondo T, Hara T, Kosaka N (2003) Use of 18F-choline and $11 \mathrm{C}$-choline as contrast agents in positron emission tomography imaging-guided stereotactic biopsy sampling of gliomas. J Neurosurg 99:474-479

8. Alongi P, Quartuccio N, Arnone A et al (2020) Brain PET/CT using prostate cancer radiopharmaceutical agents in the evaluation of gliomas. Clin Transl Imaging. https://doi.org/10.1007/ s40336-020-00389-7

9. Grech-Sollars M, Vaqas B, Thompson G et al (2017) An MRS- and PET-guided biopsy tool for intraoperative neuronavigational systems. J Neurosurg 127:812-818. https://doi. org/10.3171/2016.7.JNS16106

10. Louis DN, Perry A, Reifenberger G et al (2016) The 2016 World Health Organization classification of tumors of the central nervous system: a summary. Acta Neuropathol 131:803-820. https ://doi.org/10.1007/s00401-016-1545-1

11. Grech-Sollars M, Ordidge KL, Vaqas B et al (2019) Imaging and tissue biomarkers of choline metabolism in diffuse adult glioma: 18F-Fluoromethylcholine PET/CT, magnetic resonance spectroscopy, and choline kinase $\alpha$. Cancers (Basel). https://doi. org/10.3390/cancers 11121969

12. Li W, Ma L, Wang X et al (2014) (11)C-choline PET/CT tumor recurrence detection and survival prediction in post-treatment patients with high-grade gliomas. Tumour Biol 35:1235312360. https://doi.org/10.1007/s13277-014-2549-x 
13. Takenaka S, Asano Y, Shinoda J, et al (2014) Comparison of (11)C-Methionine, (11)C-Choline, and (18)F-Fluorodeoxyglucose-PET for distinguishing glioma recurrence from radiation necrosis. Neurol Med Chir (Tokyo) 54(4):280-289. https://doi. org/10.2176/nmc.oa2013-0117
Publisher's Note Springer Nature remains neutral with regard to jurisdictional claims in published maps and institutional affiliations. 